\title{
SCIENTIFIC REP

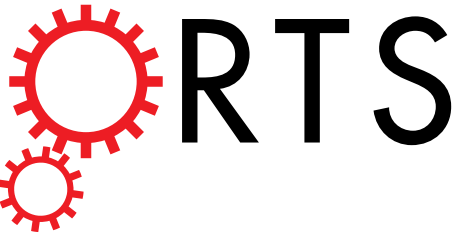

\section{OPEN Towards a gene regulatory network shaping the fins of the Princess cichlid}

Received: 19 January 2018

Accepted: 1 June 2018

Published online: 25 June 2018

\section{Ehsan Pashay Ahi $₫$ \& Kristina M. Sefc}

Variation in fin shape and size contributes to the outstanding morphological diversity of teleost fishes, but the regulation of fin growth has not yet been studied extensively outside the zebrafish model. A previous gene expression study addressing the ornamental elongations of unpaired fins in the African cichlid fish Neolamprologus brichardi identified three genes (cx43, mmp9 and sema3d) with strong and consistent expression differences between short and elongated fin regions. Remarkably, the expression patterns of these genes were not consistent with inferences on their regulatory interactions in zebrafish. Here, we identify a gene expression network (GRN) comprising $c \times 43, m m p 9$, and possibly also sema3d by a stepwise approach of identifying co-expression modules and predicting their upstream regulators. Among the transcription factors (TFs) predicted as potential upstream regulators of $11 \mathrm{co}-$ expressed genes, six TFs (foxc1, foxp1, foxd3, myc, egr2, irf8) showed expression patterns consistent with their cooperative transcriptional regulation of the gene network. Some of these TFs have already been implicated in teleost fish fin regeneration and formation. We particularly discuss the potential function of $f \circ x 3$ as driver of the network and its role in the unexpected gene expression correlations observed in N. brichardi.

The developmental mechanisms underlying fin formation in fish display remarkable similarities with those involved in appendage development in other vertebrates ${ }^{1-3}$. The ability of teleost fish to completely regenerate amputated fins from adult, differentiated cells provides a fascinating opportunity to investigate the molecular mechanisms of tissue regeneration in higher vertebrates ${ }^{4}$. Buoyed by the availability of mutants for teleost models (primarily zebrafish), studies of fin morphogenesis have elucidated molecular details about underlying genetic factors and signaling pathways 5 . In comparison, however, much less is known about the genetic factors involved in the natural morphological variation of fin shape in teleost fishes.

The fin of teleost fish is comprised of bifurcated structures, termed fin rays or lepidotrichia, which include vascularized and innervated mesenchyme enclosed by bony segments and several epidermal layers. The fin rays are connected by inter-ray tissue which also contains mesenchyme surrounded by epidermis. The fin growth is the result of the distal addition of segments through cell proliferation, differentiation and survival ${ }^{6}$. A partially amputated fin is capable of regeneration through the formation of a highly proliferative tissue (blastema) at the distal end of rays and inter-ray tissue. Despite the simple structural properties, fin regeneration involves complex processes of dedifferentiation of cells into blastema, proliferation of undifferentiated blastemal cells towards the distal end and differentiation of blastemal cells in the proximal end of the regenerating tissue ${ }^{1,7}$. At the molecular level, both ontogenetic and regenerative fin growth are tightly regulated by several interconnected signaling pathways and their downstream effectors ${ }^{6-8}$.

Extensive research has been launched to identify genes underlying fin growth and regeneration with a strong focus on the caudal fin of the zebrafish model Danio rerio ${ }^{2,8-10}$. It is only in recent years that the molecular basis of the morphological diversity of fins within and across species has attracted some attention ${ }^{11-15}$. Studies capitalizing on the natural variation in fin morphology addressed, for instance, the ventral elongation of the caudal fin in swordtail fish ${ }^{11}$, interspecific divergence in pectoral fin morphology in cichlids from Lake Malawi ${ }^{13}$ and the twin-tail phenotype of goldfish ${ }^{14}$. Here, we are interested in the molecular basis of fin filaments, that is, ornamental elongations of fins which are displayed by numerous fish species across various taxonomic groups. In our study species, the African cichlid fish N. brichardi, the unpaired fins of both males and females are conspicuously adorned by such filamentous elongations (Fig. 1A). In a previous study, we hypothesized that positional 


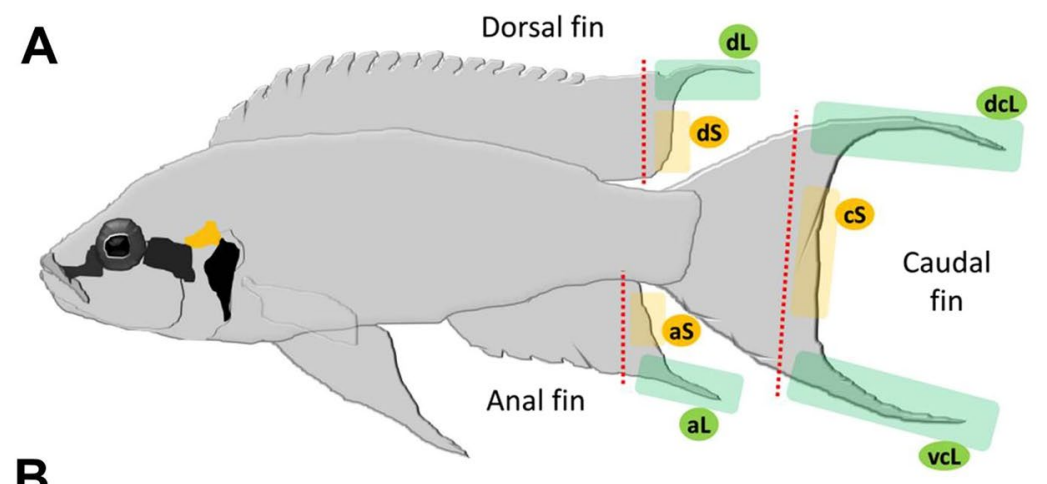

B
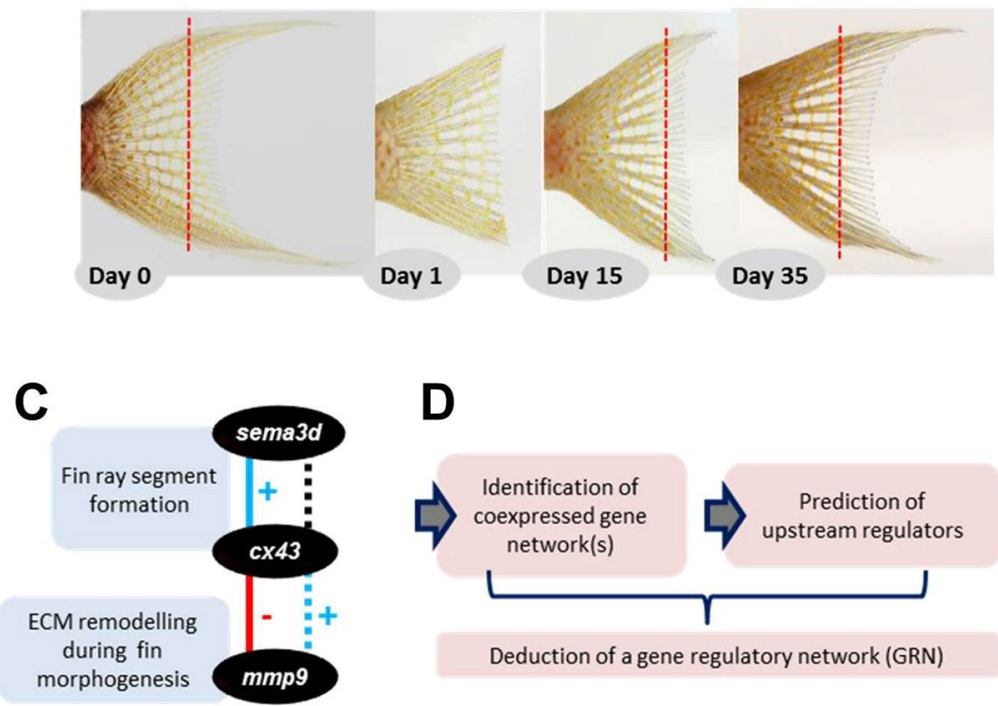

D

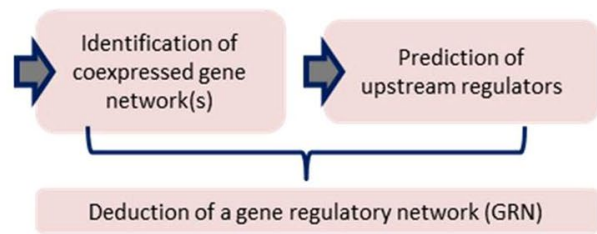

Figure 1. Fin dissections and a workflow for identifying gene regulatory network(s) underlying elongated fin phenotype in Neolamprologus brichardi. (A) An adult Lake Tanganyika cichlid fish, N. brichardi, displays filamentous elongations of the unpaired fins. Green shaded areas mark the elongated regions of the dorsal fin $(\mathrm{dL})$, anal fin $(\mathrm{aL})$, and the ventral and dorsal regions of the caudal fin ( $\mathrm{dcL}$ and $\mathrm{vcL}$ ); yellow shaded areas mark the short regions in the dorsal fin (dS), anal fin (aS), and in the center of the caudal fin (cS). The red dashed line represents the cutting line for the biopsy. (B) Tissue sampling at day 0 (first cut), day 15 (second cut) and day 35 (third cut) using the example of the caudal fin (photos by Wolfgang Gessl (www.pisces.at)). (C) Genes found to be associated with the elongated fin phenotype of $N$. brichardi, their function in zebrafish (blue squares) and their positive or negative expression correlation in zebrafish (continuous blue and red lines, respectively), as well as their contrasting expression correlation in N. brichardi (dashed lines; black colour indicates no expression correlation). (D) Schematic representation of the steps involved in the deduction of the gene regulatory network based on zebrafish co-expression data for sema3d, cx43 and mmp9.

differences in gene expression levels underlie the extreme elongation of filaments, and tested a series of candidate genes involved in fin formation and regeneration for differential expression between elongated (L) and regular (i.e. short, S) fin regions ${ }^{15}$. Comparing gene expression levels between $\mathrm{L}$ and $\mathrm{S}$ tissue sampled from both intact and regenerating fins, we detected several genes with either higher L-expression or higher S-expression ${ }^{15}$. Particularly strong and consistent signals were obtained for $c x 43 /$ Gjal and $m m p 9$, both showing elevated L-expression, and sema3d with elevated S-expression. $c x 43$ encodes for a subunit of the gap junction protein complex ${ }^{16}, m m p 9$ produces a matrix remodelling enzyme $e^{8,17}$ and sema $3 d$ encodes a conserved secreted ligand of several cell surface receptors involved in nervous system development, cell differentiation and bone homeostasis ${ }^{18,19}$. In zebrafish, sema3d functions downstream of $c x 43$ in a common pathway regulating cell proliferation and joint formation, since a knockdown of $c x 43$ results in reduced expression of sema $3 d^{18}$. Also in zebrafish, reduced expression of $c x 43$ was associated with up-regulation of $m m p 9$ in the caudal fin ${ }^{20}$. Conversely, in N. brichardi, we found no correlation between $c x 43$ and sema3d expression, whereas the expression of $c x 43$ was positively correlated with $m m p 9$ expression in each of the unpaired fins ${ }^{15}$. We also found sema3d expression to be positively correlated with the expression of a ligand of Wnt signaling pathway ( $w n t 5 b)$ across all fins, which suggested its transcriptional regulation by this pathway, consistent with findings in zebrafish neural crest cells ${ }^{21}$. Interestingly, the elevated expression of $c x 43$ in the elongated fin regions of $N$. brichardi was not accompanied by an increase of the length of the fin ray segments, which contrasts with the effects of $c x 43$ manipulation in zebrafish ${ }^{16,22}$. A recent study, however, suggests diverse functions of $c x 43$ during zebrafish skeletal growth and different mutants of $c \times 43$ can 
confer distinct bone phenotypes in fin and vertebrae ${ }^{23}$, though the underlying gene regulatory networks have yet to be elucidated.

The expression patterns observed in our previous study raise the possibility that in the unpaired fins of $N$. brichardi, mmp9 and $c x 43$ belong to a co-expression network regulated by shared upstream player(s). It is worth emphasizing that the inter-dependent functional modulation of cx 43 and mmp enzymes and their coexpression have attracted considerable attention due to the high abundance and diverse functions of these enzymes in various tissues ${ }^{24}$. However, little is known about their transcriptional co-regulation, particularly in the context of skeletal morphogenesis. The lack of an expression correlation between sema3d and $c x 43$ suggests their regulatory decoupling and the potential involvement of a distinct upstream effector for sema3d during fin morphogenesis in $N$. brichardi, in contrast to evidence from zebrafish ${ }^{18}$. Alternatively, sema3d might pertain to the same regulatory network as $c x 43$ and $m m p 9$, but its expression might be repressed by certain regulator(s) of the network. In this study we sought to identify gene regulatory networks (GRN) comprising $c x 43, m m p 9$, and sema3d using a stepwise approach of identifying co-expression module(s) and predicting their upstream regulators ${ }^{25}$ (Fig. 1D). Candidate genes were identified from co-expression data available for zebrafish and tested for co-expression with $m m p 9, c x 43$ and sema3d in the intact and regenerating fin tissue of $N$. brichardi. The prediction of transcription factors for genes of interest was based on the annotated genome of the Nile tilapia. Among the transcription factors (TFs) predicted as potential upstream regulators of 11 co-expressed genes, six TFs showed an expression pattern consistent with their cooperative transcriptional regulation of the gene network. In particular, one of the TFs, foxd3, may underlie the observed expression patterns of $c x 43, m m p 9$ and sema3d in N. brichardi. Thus, we provide the first evidence for a potential GRN comprising $c x 43, m m p 9$ and sema3d, as well as several other genes with unknown roles in fin formation, and provide a basis for further functional investigations in model and non-model organisms.

\section{Results}

Expression analysis of candidate genes co-expressed with $m m p 9, c x 43$ and sema3d. In order to identify gene co-regulatory network(s) involved in the outgrowth of filaments on the unpaired fin of N. brichardi, we conducted stepwise candidate gene selection (described by Ahi et al. ${ }^{25}$ ) using a zebrafish co-expression database, COXPRESdb ${ }^{26}$. Our previous study suggested the genes $m m p 9, c x 43$ and sema3d to be associated with the fin phenotype, based on expression differences between elongated and short fin tissue ${ }^{15}$. For each of these genes, we selected 8-9 strongly co-expressed genes from the zebrafish database (Supplementary data 1). Two of these genes, $b m p 4$ and junb, had already been included as candidate genes in the previous study ${ }^{15}$, such that 23 new candidate genes were inferred at this step. We tested the expression of these genes in the intact fin tissue (stage 0 ) and at two stages during regeneration (stages 1 and 2). In the following text 'expression in the elongated region' is abbreviated as 'L-expression, and reported as 'higher' or 'lower' in comparison to expression in the short region ('S-expression'). In our previous study, L-expression of $m m p 9$ and $c x 43$ was higher and L-expression of sema3d was lower than their respective S-expression ${ }^{15}$. For 14 of the 23 tested genes, linear mixed models detected significant $\mathrm{L} / \mathrm{S}$ expression differences (after Bonferroni correction for multiple testing), some of which were confounded by significant interactions with fin type and developmental stage (Supplementary data 2). Based on post-hoc tests (paired t-tests) used to identify L/S differences that were replicated across fin types and across developmental stages, we then identified 9 genes ( 3 genes for each set of the candidates co-expressed with $m m p 9, c x 43$ and sema3d) with significant L/S expression differences in at least two stages of at least two fins (genes marked yellow in Figs 2-4; Supplementary data 2).

All of these genes had higher L-expression, although not necessarily in each fin or each regeneration stage (e.g. mab21l1, txn and angptl5; Figs 2-4). Noteworthy, the expression correlations of the 9 genes with L/S expression differences and the genes on the basis of which they were selected for the analysis (i.e., $m m p 9, c x 43$ or sema3d) were positive in the zebrafish database. Therefore, higher L-expression was expected for genes associated with $m m p 9$ and $c x 43$, whereas the genes selected on the basis of their co-expression with sema3d in zebrafish were expected to behave in the opposite way (i.e. lower L-expression, consistent with sema3d). Indeed, the 6 genes co-expressed with $m m p 9$ and $c x 43$ displayed higher L-expression (genes marked yellow in Figs 2 and 3), but opposed to expectations based on zebrafish expression data, the 3 genes co-expressed with sema3d showed opposite $\mathrm{L} / \mathrm{S}$ expression differences to sema3d (i.e. again higher L-expression).

Based on the above data, we combined those genes, which showed consistent $\mathrm{L} / \mathrm{S}$ expression patterns (i.e. consistent higher L-expression), into modules (module 1: mmp9-anxa2a-cd63-txn, module 2: $c x 43-d p y s l 5 a-p f k p a$, and module 3: angptl7-c1qtnf5-angptl5; mab21l1 was ignored due to its inconsistent expression pattern), which were used to select additional candidate genes in order to extend our potential network. For each module, we selected five genes which were co-expressed with all the genes in the module according to the zebrafish database (Supplementary data 1), and tested them for L/S expression differences in the N. brichardi fins as described above (Supplementary data 2). This led to the identification of three more genes with L/S expression differences in almost all stages and fins derived from the first and second module (genes marked blue in Figs 2 and 3 ), whereas no L/S expression difference was detected in the genes derived from the third module. Two genes, csrpla and gnaola, had higher L-expression, and kif5a showed lower L-expression. We also note that the expression of gnaola was very stable across regeneration stages (Fig. 3; Supplementary data 2). After the two steps of gene selection described above, a total of 10 genes ( 8 in the first step, 2 in the second step) were found to have consistently higher L-expression, as previously found for $c x 43$ and $m m p 9$. Positive expression correlations in most pairwise comparisons among these genes suggested that they could pertain to a co-regulated gene network with shared upstream transcriptional regulators.

Prediction of upstream regulators. In order to maximize the power of our approach, the following step was based on genes with congruent L/S expression differences (i.e., higher L-expression). We searched 

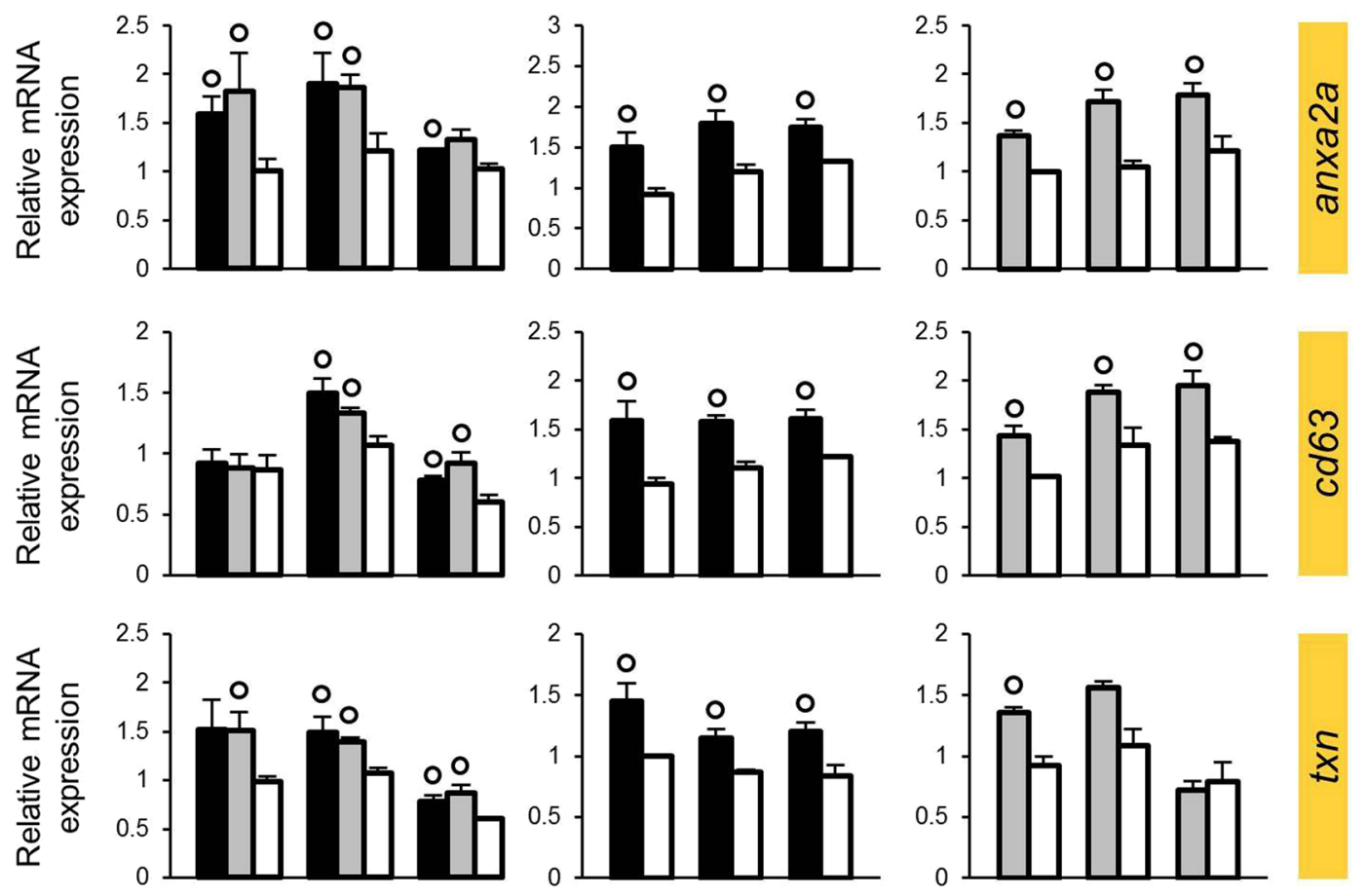

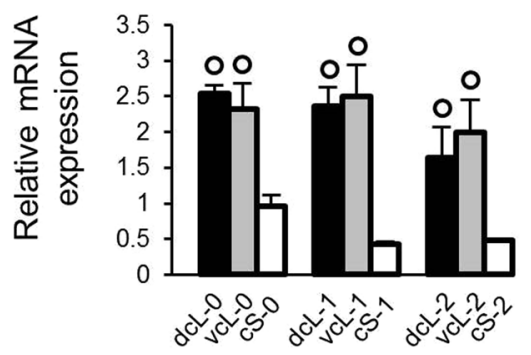

Fin regions

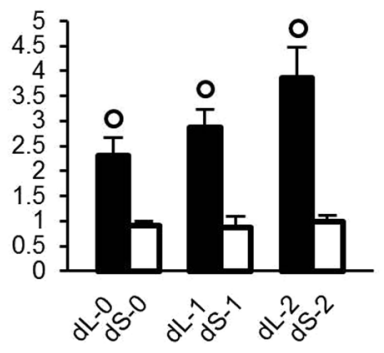

Fin regions

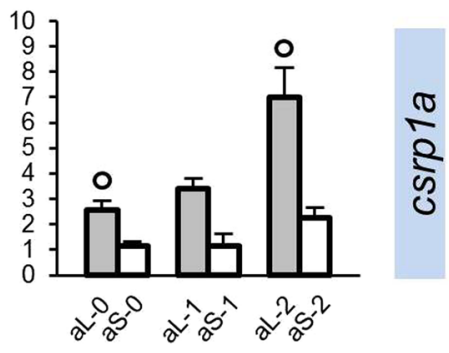

Fin regions

Figure 2. Expression levels of candidate genes selected based on co-expression with $m m p 9$. Means and standard deviations of RQ in three biological replicates are shown for the elongated (L) and short (S) regions of the caudal, dorsal and anal fin in original (stage 0 ) and regenerating tissue. See Fig. 1A for fin region codes; numbers 0 to 2 identify regeneration stages. Circles above bars indicate significantly elevated expression $(\mathrm{P}<0.05$ in paired t-tests) in comparisons between $\mathrm{L}$ and $\mathrm{S}$ tissue samples (i.e., compared to the bar matching the shade of the circle); note that the analysis was restricted to comparisons within the same fin type and the same regeneration stage. Genes highlighted in yellow and blue were identified in the first and second step of our gene selection procedure, respectively.

for potential upstream regulators of the identified gene network through prediction of TF binding sites in the upstream regulatory sequences of $c x 43, m m p 9$ and the 10 new genes with consistently higher L-expression (i.e. mab21l1 was dropped due to its inconsistent expression pattern; sema3d and kif5a because of their higher S-expression). We found more than 30 motifs present in the regulatory sequences of at least half of the genes (Supplementary data 1). By parsing the motifs against the vertebrate TF binding sites, we compiled a list of top matched TFs for each motif (Supplementary data 1). After analysing the expression levels of the 13 most significantly enriched TFs predicted by two different algorithms in the fins of N. brichardi, we found six TFs displaying L/S differential expression (Fig. 5; Supplementary data 2). Two of these TFs, foxc1 and foxp1, showed lower L-expression, whereas the others showed higher L-expression. The most consistent differential expression was observed in foxd3, with higher L-expression across all fins and stages. Finally, we checked whether these 6 TFs had binding sites in the upstream regions of sema $3 d$ and kif $5 a$, i.e. the two candidate genes with $\mathrm{S}>\mathrm{L}$ expression. Indeed, binding sites for foxd 3 , foxc 1 and irf 8 are present upstream of sema3d, and irf 8 also has a binding site upstream of kif5a. Taken together, these findings implicate the involvement of several TFs in regulating the co-expression network through potential cooperative interaction(s) and short distance cis-binding promoter activity. Furthermore, foxd 3 appeared to be an upstream candidate for transcriptional induction of $c x 43$ and $m m p 9$ while possibly acting as a transcriptional repressor of sema3d in N. brichardi fins. 

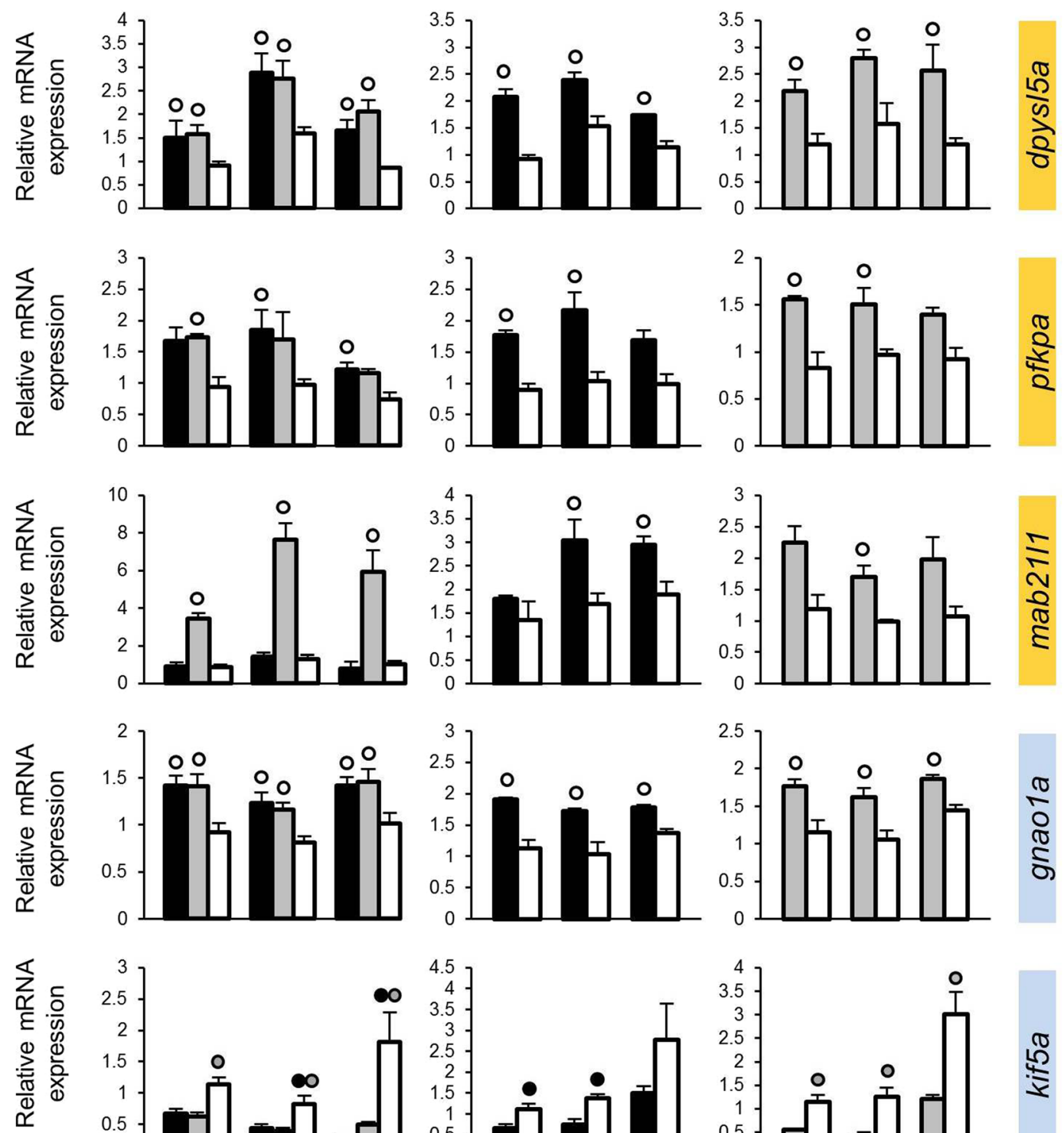

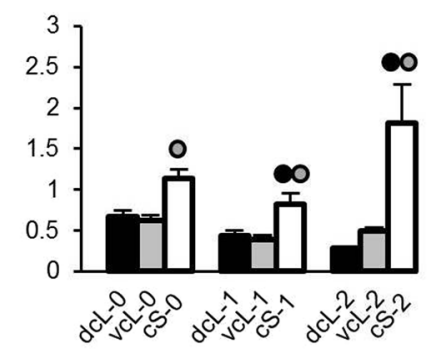

Fin regions

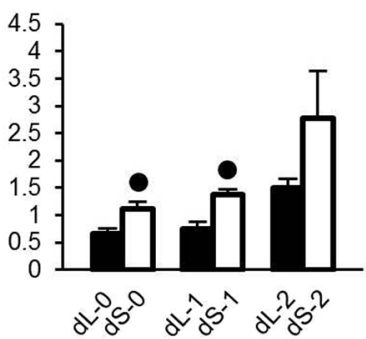

Fin regions

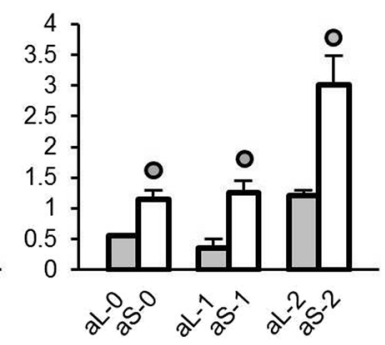

Fin regions

Figure 3. Expression levels of candidate genes selected based on co-expression with $c x 43$. Means and standard deviations of RQ in three biological replicates are shown for the elongated (L) and short (S) regions of the caudal, dorsal and anal fin in original (stage 0) and regenerating tissue. See Fig. 1A for fin region codes; numbers 0 to 2 identify regeneration stages. Circles above bars indicate significantly elevated expression $(\mathrm{P}<0.05$ in paired $\mathrm{t}$-tests) in comparisons between $\mathrm{L}$ and $\mathrm{S}$ tissue samples (i.e., compared to the bar matching the shade of the circle); note that the analysis was restricted to comparisons within the same fin type and the same regeneration stage. Genes highlighted in yellow and blue were identified in the first and second step of our gene selection procedure, respectively.

Expression correlations. Pairwise expression correlation analyses among the investigated genes, i.e. TFs and the candidate network genes, used data pooled across all fins (Fig. 6) as well as for each fin separately (Fig. S1). Almost all genes of the putative co-expression network (except angptl7) showed positive expression correlations with rest of the members in data pooled across fins (blue shadings in Fig. 6A). sema3d displayed negative expression correlations with most members of the network and kif5a showed negative correlations with five members (red shadings in Fig. 6A). Some of the expression correlations between the genes (mainly positive ones) were detected in each of the fins (yellow numbers in Fig. 6A).

TFs varied in the number of co-expressed network genes, which suggests that some TFs participate in the regulation of more network genes than others. In particular, foxc1 and foxpleach showed a high number of negative correlations, indicating a potential repressive regulatory role of foxc1 and foxp 1 on the transcription of the gene network. Interestingly, foxc1 had a positive expression correlation with sema3d. The remaining TFs, most 

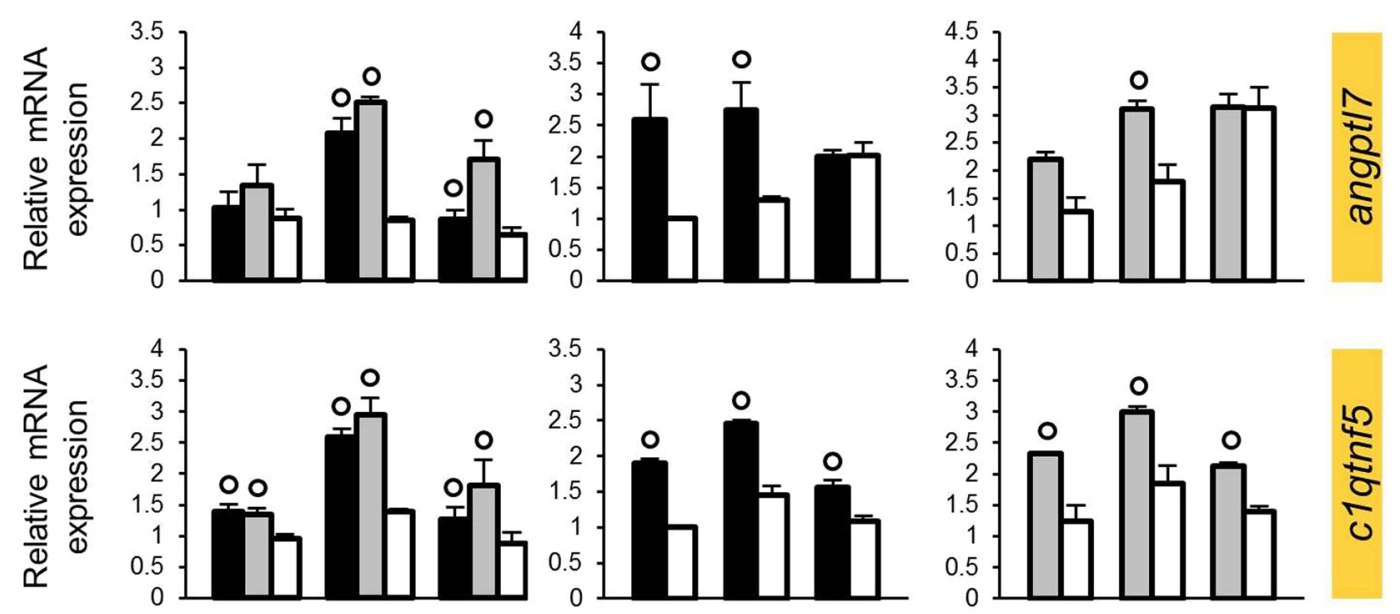

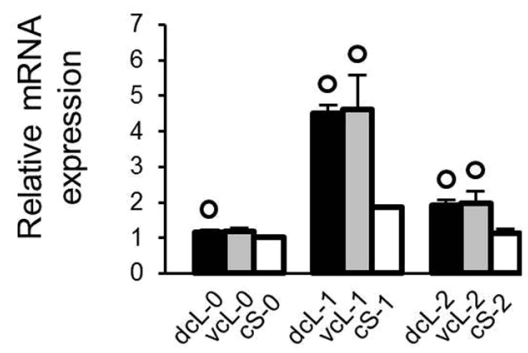

Fin regions

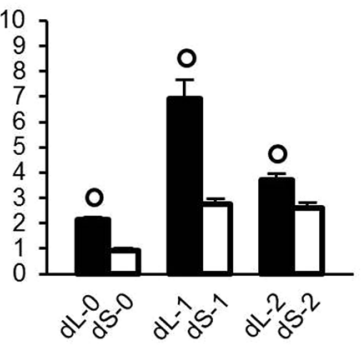

Fin regions

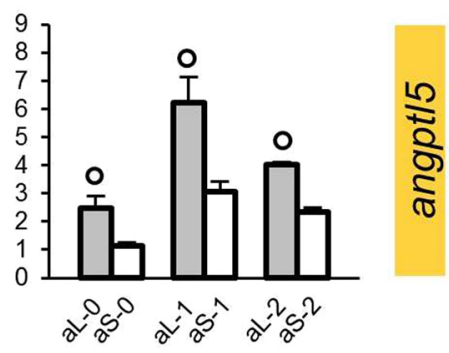

Fin regions

Figure 4. Expression levels of candidate genes selected based on co-expression with sema3d. Means and standard deviations of RQ in three biological replicates are shown for the elongated (L) and short (S) regions of the caudal, dorsal and anal fin in original (stage 0 ) and regenerating tissue. See Fig. 1A for fin region codes; numbers 0 to 2 identify regeneration stages. Circles above bars indicate significantly elevated expression $(\mathrm{P}<0.05$ in paired t-tests) in comparisons between $\mathrm{L}$ and $\mathrm{S}$ tissue samples (i.e., compared to the bar matching the shade of the circle); note that the analysis was restricted to comparisons within the same fin type and the same regeneration stage. Genes highlighted in yellow and blue were identified in the first and second step of our gene selection procedure, respectively.

notably foxd 3 and $m y c$, had positive correlations with numerous network genes, suggesting a potential inductive regulatory role of these TFs on transcription of the gene network. Again, correlations of TFs with sema3d were in the opposite direction, i.e. negative (egr 2 and foxd3).

Many of the expression correlations between TFs and the network genes, which were detected when data were pooled across all fins, were not observed in each of the individual unpaired fins (Fig. 6A, Fig. S1), perhaps due to reduced statistical power. Noteworthy exceptions are consistent correlations of foxd 3 and egr 2 with five and two network genes, respectively.

\section{Discussion}

The identification of gene regulatory networks (GRNs) represents an exciting research avenue in the exploration of phenotypic variation. Based on resources established in model species, we can now investigate changes in GRNs in relation to the tremendous morphological diversity across non-model organisms and track down the molecular mechanisms behind morphological diversification ${ }^{27-29}$. In non-model teleost fishes, for instance, recent efforts targeted GRNs involved in the morphological variation of different skeletal structures ${ }^{30-33}$. The present study addresses the regulation of fin shape and draws upon correlated expression patterns and predicted regulatory interactions to identify members of a GRN associated with the elongated fin filaments displayed by the East African "Princess cichlid", N. brichardi.

In a previous gene expression study of fin growth and shape in $N$. brichardi, we identified three genes $c x 43$, $m m p 9$ and sema3d that were differentially expressed between short and long regions in both intact and regenerating unpaired fins. As explained in the introduction, the expression patterns of these genes in N. brichardi were not consistent with inferences on their regulatory interactions in zebrafish ${ }^{18}$. In particular, our data raised the possibility that sema3d is either regulated independently from $c x 43$ and mmp9 in N. brichardi, or that its expression in the context of a shared network is modulated by additional regulators. Furthermore, the expression of $c \times 43$ was positively correlated with $m m p 9$ expression in $N$. brichardi fins ${ }^{15}$, whereas reduced expression of $c x 43$ was associated with up-regulation of $m m p 9$ in the zebrafish caudal $\mathrm{fin}^{20}$. We also note that in contrast to zebrafish, where esco2 functions as upstream transcriptional regulator of $c x 43$ and sema3 $d^{34}$, data in $\mathrm{N}$. brichardi did not support 

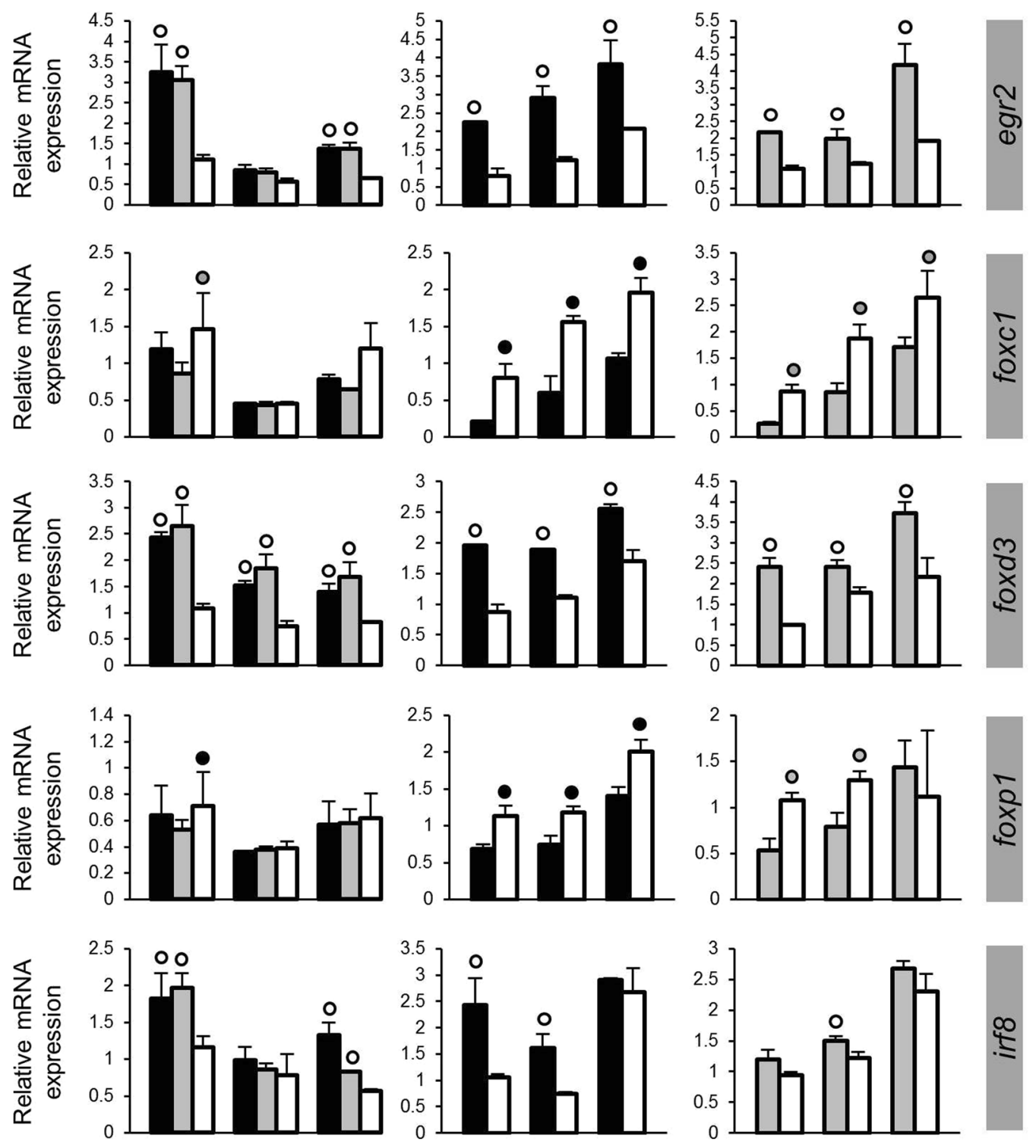

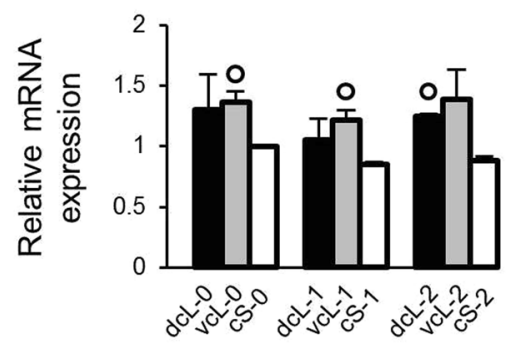

Fin regions

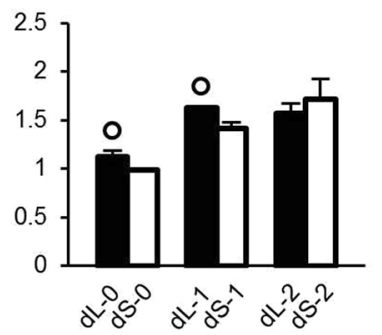

Fin regions

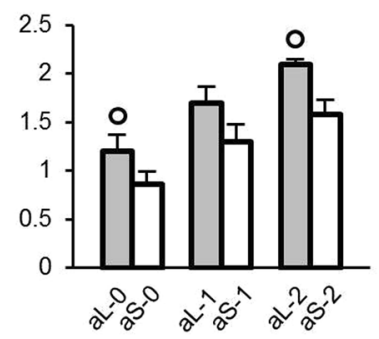

Fin regions

Figure 5. Expression levels of predicted upstream regulators. Means and standard deviations of RQ in three biological replicates are shown for the elongated (L) and short (S) regions of the caudal, dorsal and anal fin in original (stage 0 ) and regenerating tissue. See Fig. $1 \mathrm{~A}$ for fin region codes; numbers 0 to 2 identify regeneration stages. Circles above bars indicate significantly elevated expression $(\mathrm{P}<0.05$ in paired t-tests) in comparisons between $\mathrm{L}$ and $\mathrm{S}$ tissue samples (i.e., compared to the bar matching the shade of the circle); note that the analysis was restricted to comparisons within the same fin type and the same regeneration stage.

a regulatory link between $e s c o 2$ and these genes ${ }^{15}$. In order to elucidate the regulatory background of $c x 43, m m p 9$ and sema 3 and investigate the potential divergence in regulatory mechanisms between taxa, we identified a regulatory network through the assembly of co-expression modules and prediction of their upstream regulators. Furthermore, we deduced a possible scenario explaining the discrepancy of sema3d expression between zebrafish 
A

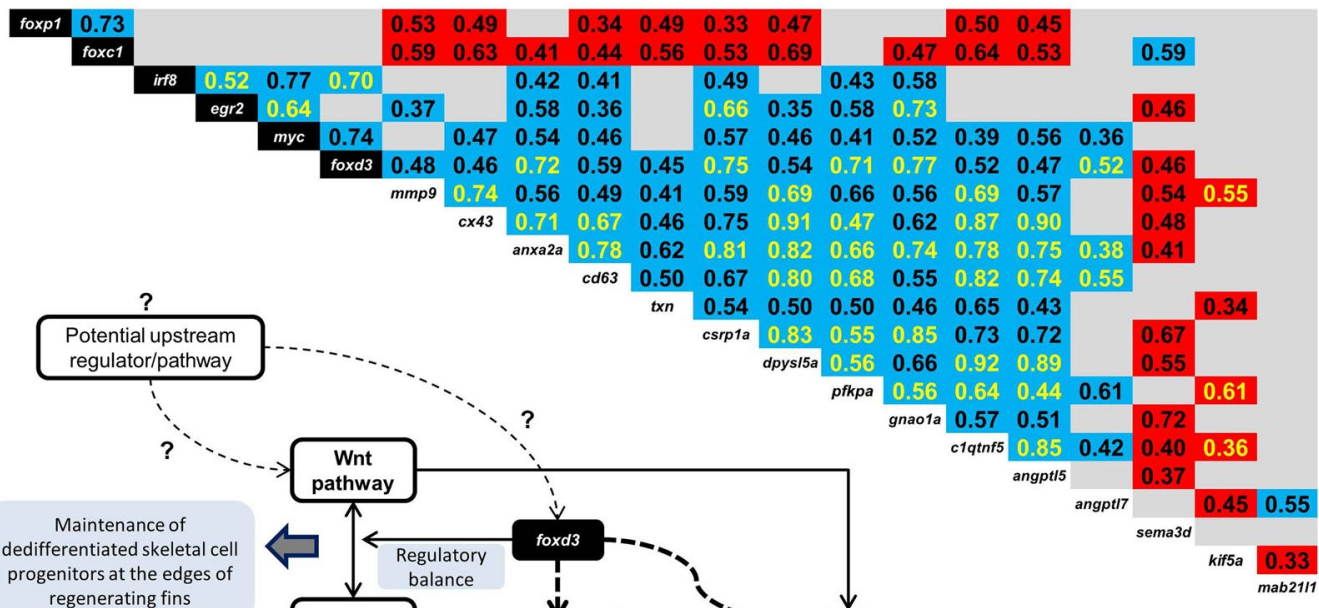

B

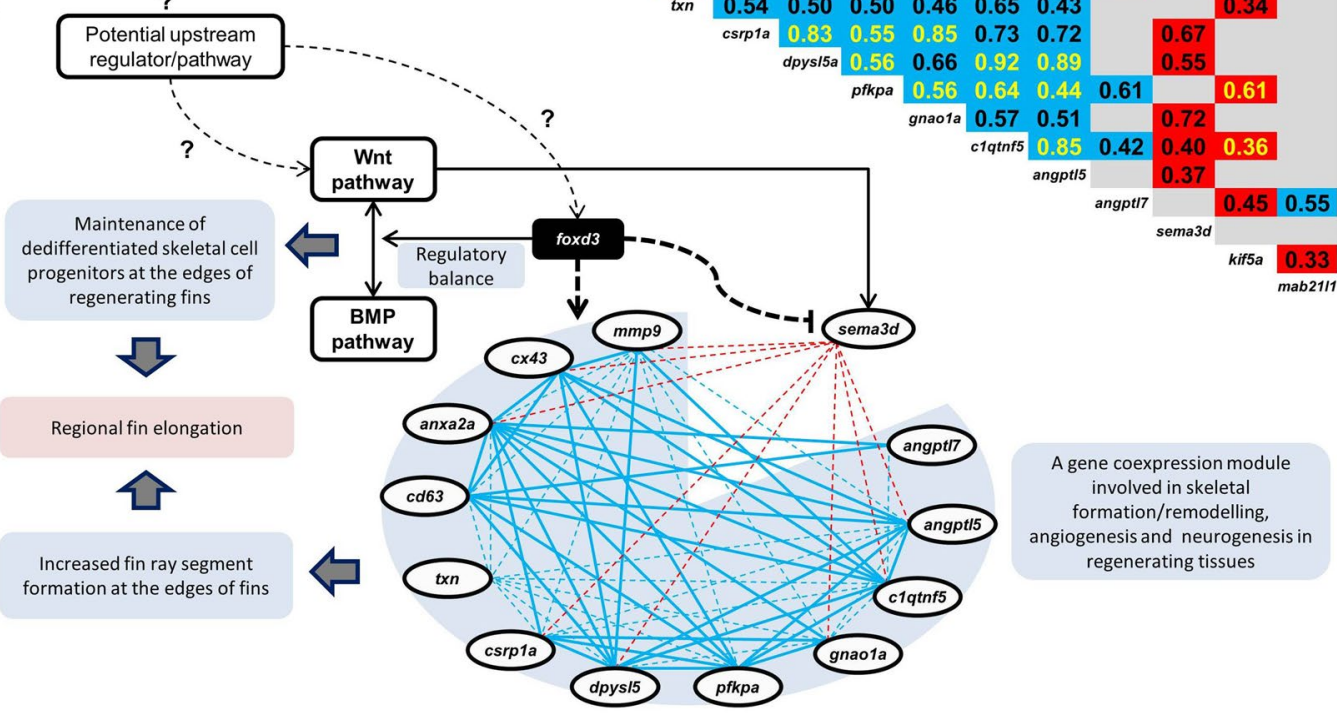

Figure 6. A proposed gene regulatory network underlying fin shape elongation in Neolamprologus brichardi. (A) Significant expression correlations between members of a gene network and their predicted upstream regulators across the unpaired fins of $N$. brichardi. Numbers indicate Pearson correlation coefficients $(\mathrm{P}<0.01$ in 2-tailed tests) based on gene expression data pooled across fins. Blue and red shadings represent positive and negative expression correlations, respectively. Yellow font indicates expression correlations, which were also significant in each of the three fins when analysed separately. (B) A proposed gene interaction model linking the identified genes and their functions, as well as their role in fin morphogenesis and regeneration in N. brichardi. In the co-expression module, positive (blue lines) and negative (red lines) expression correlations are indicated by solid lines, if the correlation was detected in each of the unpaired fins, while fin-specific correlations are indicated by dashed lines. Previously described regulatory connections are represented by black solid lines. Potential transcriptional induction and repression inferred in the present study is depicted by dashed black lines, and questionmarks indicate potential upstream regulatory connections which are not investigated in this study.

and N. brichardi, in which an identified TF, foxd3, could act as transcriptional activator of $c x 43$ and $m m p 9$ and repressor of sema3d in N. brichardi.

Although the results of our previous study already suggested divergence in regulatory mechanisms between zebrafish and N. brichardi, the lack of data in other species necessarily restricted our search for co-expressed candidate genes to the zebrafish database. This reduces the power of our approach inasmuch as genes, which are part of the GRN in $N$. brichardi but show no expression correlations in zebrafish, will not be included among the set of candidate genes. Despite this limitation, the approach proved successful and led to the identification of a module of 10 genes with correlated expression and consistent L/S expression differences, suggesting their co-regulation through shared regulator(s) during fin formation in N. brichardi. Notably, the co-expression based approach to candidate gene selection yielded a similar proportion of genes with consistent L/S expression differences ( 10 out of 38 tested genes) as we had achieved in our previous study ${ }^{15}$, where candidate genes were carefully selected based on their known role in fin development, morphogenesis and/or regeneration in the zebrafish (13 out of 40 tested genes). The similar success rates suggest that after exploiting existing knowledge of gene function for the selection of candidate genes, stepwise co-expression-based candidate gene selection is indeed an efficient approach to extend the set of promising candidate genes ${ }^{25,35}$.

Some of the genes detected in the present study have already been implicated in studies of teleost fish fin regeneration and morphogenesis (see details in Table 1). These include anxa2a, a member of the annexin family $^{36}$, two angiopoietic protein encoding genes, angptl5 and angptl $7^{11,37}, d p y s l 5 a$, which encodes a member of the Collapsin response mediator protein (CRMP) family ${ }^{38}$, and clqtnf5, encoding a basement membrane component ${ }^{39}$. Some other members of the gene network are not directly indicated in fin regeneration but appeared to have related functions in vertebrates. For instance, csrpla, encoding a member of the cysteine-rich protein family, is required for neuron regeneration capability of adult zebrafish ${ }^{40}$, and $t x n$ and $c d 63$ are expressed during neural 


\begin{tabular}{|c|c|c|c|}
\hline \multicolumn{2}{|c|}{ Gene functions/expression patterns } & \multirow{2}{*}{\begin{tabular}{|l} 
Species \\
Zebrafish \\
Xenopus
\end{tabular}} & \multirow{2}{*}{\begin{tabular}{|l|} 
References \\
36,77
\end{tabular}} \\
\hline anxa2a & $\begin{array}{l}\text { Up-regulated in regenerating fin and limb; represses histone methylation factors involved } \\
\text { in epigenetic regulation of caudal fin regeneration }\end{array}$ & & \\
\hline angptl5 & Involved in angiogenesis and up-regulated during elongation of caudal fin & Swordtail fish & 11 \\
\hline angptl7 & Involved in angiogenesis and blastema formation in regenerating fin & Medaka fish & 37 \\
\hline$d p y s l 5 a$ & Involved in neural development and up-regulated in blastema of regenerating caudal fin & Zebrafish & 38 \\
\hline clqtnf5 & $\begin{array}{l}\text { A downstream target of Aryl hydrocarbon pathway during fin regeneration, a pathway } \\
\text { inhibiting fin regeneration }\end{array}$ & Zebrafish & 39 \\
\hline csrpla & Required for neuron regeneration capability & Zebrafish & 40 \\
\hline kif5a & An inhibitor of neuron regeneration & Mouse & 42 \\
\hline$t x n$ & Up-regulated at onset of neural regeneration and limb regeneration & $\begin{array}{l}\text { Axolotl } \\
\text { Xenopus }\end{array}$ & 41,77 \\
\hline$c d 63$ & Up-regulated at onset of neural regeneration & Axolotl & 41 \\
\hline$m y c$ & $\begin{array}{l}\text { Up-regulated at onset of neural regeneration, and in blastema of regenerating fin, limb and } \\
\text { lens }\end{array}$ & $\begin{array}{l}\text { Zebrafish } \\
\text { Axolotl } \\
\text { Xenopus }\end{array}$ & $41,51-53$ \\
\hline $\operatorname{irf8}$ & $\begin{array}{l}\text { Involved in macrophage activation; indirectly participates in cell proliferation, growth and } \\
\text { survival during fin regeneration }\end{array}$ & Zebrafish & 55,56 \\
\hline foxd3 & $\begin{array}{l}\text { Involved in repression of Wnt pathway in bone regeneration, and up-regulated in caudal fin } \\
\text { elongation }\end{array}$ & $\begin{array}{l}\text { Human } \\
\text { Swordtail fish }\end{array}$ & 11,65 \\
\hline \multicolumn{4}{|c|}{ Regulatory connections } \\
\hline egr2-foxd3- myc & $\begin{array}{l}\text { foxd } 3 \text { represses egr } 2 \text { expression while its expression requires } m y c \text { transcription during } \\
\text { neural crest formation }\end{array}$ & Xenopus & 78,79 \\
\hline egr2-myc & Transcriptional induction of egr 2 by $m y c$ in apoptotic fibroblasts & Mouse & 80 \\
\hline$m y c-i r f 8$ & Antagonizing $m y c$ mediated transcriptional repression by irf8 in activated macrophage & Mouse & 50 \\
\hline foxc1-foxp1 & $\begin{array}{l}\text { Co-regulation of cardiac muscle differentiation and transcriptional regulation of foxp } 1 \text { by } \\
\text { foxc1 in hair follicle stem cells }\end{array}$ & $\begin{array}{l}\text { Mouse } \\
\text { Human }\end{array}$ & $81-83$ \\
\hline
\end{tabular}

Table 1. Functions and expression patterns in appendage regeneration and morphogenesis, and regulatory connections of the identified network genes in vertebrates.

regeneration in amphibians ${ }^{41}$. On the contrary, an orthologue of kif $5 a$ in mouse, encoding a member of the kinesin family, can act as an inhibitor of neural regeneration ${ }^{42}$. Thus, decreased L- expression of kif5a and increased L-expression of $\operatorname{csp} 1 a$, txn and $c d 63$ might be indicators of faster neuronal growth in the elongated fin regions.

Six TFs with binding sites in the promoter sequences of the network genes displayed L/S differential expression consistent with the module genes, which suggests their potential cooperative transcriptional regulation of the network genes during fin morphogenesis. Three of the TFs, foxc1, foxp 1 and foxd 3 belong to a conserved Fork head (Fox) protein family which acts as activators or repressors in various ontogenetic processes such as developmental patterning and organogenesis in vertebrates ${ }^{43,44}$. The fourth $\mathrm{TF}, m y c(c-M y c)$, encodes a nuclear phosphoprotein with diverse cellular functions which is well known for its role in the reprograming of differentiated cell types into pluripotent stem cells ${ }^{45}$. The fifth TF, egr2 (Krox20), is a C2H2-type zinc-finger protein with prominent roles in hindbrain development ${ }^{46}$ and several physiological processes like bone remodelling related functions ${ }^{47-49}$. Finally, $\operatorname{irf8}(I C S B P)$ is a member of the interferon regulatory factor (IRF) family, which has a primary role in innate immunity related functions such as macrophage activation ${ }^{50}$. Regulatory interconnections between some of these TFs have already been demonstrated in other vertebrates (see details in Table 1). Data from the literature together with the expression correlations observed in this study, including the positive correlations between foxc1-foxp1, egr2-myc, and myc-foxd3, raise the possibility that one (or more) of these TFs might act upstream of the other TFs during fin formation.

Furthermore, three of these TFs, $m y c$, irf 8 and foxd 3 have been already implicated in teleost fish fin regeneration/formation (Table 1). myc is among a few TFs required for the induction of pluripotent stem cells that appeared to be expressed during fin regeneration ${ }^{51}$. In amphibians, differential regulation of $m y c$ is also reported during blastema formation in regenerating limbs and lens ${ }^{52,53}$. The function of $m y c$ during vertebrate appendage regeneration requires further investigation but may be similar to that of its ortholog in fruit fly, which potentiates regenerative growth by abrogation of cell fate commitment in regenerating wing discs ${ }^{54}$. In zebrafish larva, the knock down of irf8 causes depletion of macrophages in regenerating fins and reduces cell proliferation and growth of the regenerating fin ${ }^{55}$. It also leads to aberrant apoptosis of the regenerative cells, suggesting that macrophages support the survival of regenerative cells ${ }^{56}$. In adult zebrafish, macrophages control fin outgrowth and bony ray patterning through modulation of blastema proliferation in a stage-dependent manner ${ }^{57}$. The irf 8 mediated activity of macrophages might affect fin regeneration through the regulation of other potential players, e.g. myc and $m m p 9^{58}$ or/and crosstalk with Wnt/ $\beta$-catenin signaling ${ }^{57}$, as already characterized in mammalian cells ${ }^{59,60}$.

Perhaps the most intriguing TF identified in our study is foxd3, which showed consistently higher L-expression in all fins and stages as well as expression correlations with several of the network genes across all fins. The function of foxd 3 is well studied because of its critical role in epithelial to mesenchymal transition (EMT) of the neural crest progenitors and in maintaining their multipotency (in concert with other TFs like $m y c)^{61}$. These processes are accompanied by fine-tuned expression regulation of downstream effectors including $c \times 43$ and $m m p$ genes ${ }^{61}$. Processes reminiscent of EMT and dedifferentiation of skeletal cells play a pivotal role in the regeneration of zebrafish fins ${ }^{1,62}$. At the molecular level, opposing activities of Wnt and BMP signaling pathways coordinate the 
maintenance of dedifferentiated osteoblast progenitors at the distal tip of the regenerative blastema ${ }^{63}$. foxd 3 is known to be a downstream effector of Wnt signaling in zebrafish ${ }^{64}$, and recently, a study of bone regeneration in human has demonstrated foxd 3 dependent repression of Wnt signaling pathway ${ }^{65}$. In addition, foxd3 is required for the modulation of the balance between BMP and Wnt signals in developing neural crest derivatives in zebrafish $^{66}$. Interestingly, foxd 3 is also highly expressed in the exaggerated fin outgrowth (the sword) of male sword-tail fish $^{11}$. In our previous study, we found differential L/S expression of Wnt and BMP components in the original and regenerating fins of $N$. brichardi $i^{15}$. The above observations together with our results suggest a potential modulatory function of foxd 3 during fin elongation in N. brichardi by transcriptional regulation of the identified gene network and possible coordination of signals mediated by Wnt and BMP pathways (Fig. 6B).

Intriguingly, the regulatory role of foxd 3 might offer an explanation for the opposing L/S expression differences of $c x 43$ and sema3d in N. brichardi. Supported by findings in zebrafish, two regulatory mechanisms are possible. First, while foxd3 induces $c x 43$ expression in the elongated fin regions it might also repress sema3d expression indirectly through inhibition of Wnt pathway. Expression levels of sema3d and wnt5b were positively correlated in the fins of $N$. brichardi $i^{15}$ and Wnt has been shown to regulate sema3d in zebrafish ${ }^{21}$. Alternatively, foxd 3 might act as a direct transcriptional repressor of sema3d, given that we found a foxd 3 binding motif in the promoter sequence of sema3d. Indeed, a recent study demonstrated bimodal transcriptional activity of foxd3 (i.e. as transcriptional activator and repressor) and suggested sema3d as one of its downstream targets in zebrafish neural crest cells ${ }^{67}$. Figure 6B summarizes the proposed interactions between the identified genes and signalling pathways, which may underlie the regional elongation of unpaired fins in N. brichardi. Further functional studies are now required to confirm this regulatory mechanism during fin formation and regeneration.

\section{Conclusions}

In the present work, we linked independent findings in vertebrates (mainly fish) in order to deduce potential regulatory interactions among co-expressed candidate genes and predicted transcription factors in the framework of a gene regulatory network associated with teleost fin shape. Some of the involved genes are known to produce signalling proteins, transcription factors or structural proteins with functions in fin formation and regeneration, whereas the role of some other genes in fin shape formation is not yet evident. Functional studies are necessary to confirm the morphogenetic impact of network genes, and comparative studies across species with similar as well as contrasting fin shapes will inform on the relationship of the identified network genes with fin shape variation.

\section{Methods}

Fin sampling, RNA isolation and cDNA synthesis. The tissue samples used in this study were the same as in our previous study of candidate gene analysis where fish husbandry, tissue sampling and RNA analysis protocols are described in details ${ }^{15}$. In brief, samples were taken from 24 captive bred adult individuals of $N$. brichardi, 12 males and 12 females with total length of 5-7 cm. Prior to fin dissection, fish were anesthetized using 0.04 gram of MS-222 per litre of water and their fins were cut in front of the first ray bifurcation (branching) under a stereomicroscope (red dashed lines in Fig. 1A,B). Tissues from the elongated and the short region of each fin biopsy (green and yellow areas in Fig. 1A) were obtained and stored frozen in RNAlater (Qiagen) until RNA isolation.

Gene expression was quantified in the original tissue (stage 0 ) and twice during regeneration, including a biopsy at day 15 after the first cut, when the elongated fin tips become apparent (stage 1), and another biopsy at day 35 after the second cut, when fin elongation was near to its original size (stage 2; Fig. 1B). Corresponding tissue samples from 8 fish ( 4 males and 4 females) were pooled as biological replicates ( $\mathrm{n}=3$ replicates), and RNA isolation and cDNA synthesis was performed as described in our previous study ${ }^{15}$. In this paper, the tissues types are identified by fin type (dorsal, caudal, anal), region $(\mathrm{L}=$ elongated, $\mathrm{S}=$ short) and stage $(0,1,2)$; for instance, aS- 0 indicates the short region of the anal fin at stage 0 . In the caudal fin, the dorsal and the ventral elongated regions are specified by 'd' and 'v', respectively, such that dcL and vcL refer to the dorsal and ventral elongated regions of the caudal fin, respectively (Fig. 1A). Anaesthesia and fin biopsies were performed under permit number BMWFW-66.007/0024-wF/v/3b/2016 issued by the Federal Ministry of Science, Research and Economy of Austria (BMWFW). All methods were performed in accordance with the relevant guidelines and regulations of BMWFW.

Gene selection, Primer design and real-time qPCR. We performed a stepwise approach ${ }^{25,35}$ based $^{2}$ on co-expression data available for zebrafish, COXPRESdb (http://coxpresdb.jp/) version $6.0^{26}$, to select candidate genes co-expressed with $m m p 9, c x 43$ and sema3d (see Results). To attain a high degree of reliability, we filtered the genes co-expressed with each of the three genes by setting the Supportability score to a minimum of 1 (as described by Obayashi \& Kinoshita ${ }^{26}$ ) (Supplementary data 1). The two rounds of candidate gene selection prompted the analysis of 39 genes, which were tested for differential expression between L and S fin tissue. In order to predict the potential upstream regulators for genes that showed $\mathrm{L}>\mathrm{S}$ expression differences, we performed motif enrichment on $4 \mathrm{~kb}$ upstream sequences (promoter and $5^{\prime}$-UTR) of these genes using the annotated genome of the Nile tilapia ${ }^{68}$ and two algorithms: $\mathrm{MEME}^{69}$ and XXmotif ${ }^{70}$. The motifs that were present in the promoters of at least half of these genes were compared to position weight matrices (PWMs) from the TRANSFAC database $^{71}$ using STAMP ${ }^{72}$ to identify matching transcription factor (TF) binding sites (Supplementary data 1).

We designed the qPCR primers for candidate genes and TFs using transcriptome data of Neolamprologus brichardi $i^{73}$. The 1-to-1 orthologues were confirmed by blasting zebrafish mRNA REfSeq IDs against $N$. brichardi transcriptome in NCBI and cross-checking the top hits returned by BLAST in the Ensembl database for zebrafish and Nile Tilapia orthologues (http://www.ensembl.org). The exon/exon junctions for each gene were also deduced from the Nile Tilapia annotated genome in the Ensembl database. This enabled us to design primers on exon junctions using Primer Express 3.0 software (Applied Biosystems, Foster City, CA, USA). Primers 
were tested for self-annealing, hetero-dimers and hairpin structures with OligoAnalyzer 3.1 (Integrated DNA Technology) (Supplementary data 3). The qPCR was conducted using Maxima SYBR Green/ROX qPCR Master Mix $(2 \times)$ by following the manufacturer's instruction (Thermo Fisher Scientific, St Leon-Rot, Germany) in 96 well-PCR plates on an ABI 7500 real-time PCR System (Applied Biosystems). The experimental set-up per run followed the preferred sample maximization method ${ }^{74}$ and the qPCR runs (including a dissociation step). The primer efficiency analyses in LinRegPCR v11.0 (http://LinRegPCR.nl) ${ }^{75}$ were conducted as described in our previous study ${ }^{15}$ and the efficiencies were between $89-111(\mathrm{E} \%)$.

Data analysis. The mean Cq values of the two previously validated reference genes, $a c t b 1$ and $r p s 18^{15}$, was used as $\mathrm{Cq}_{\text {reference }}$ and the difference between Cq values $(\Delta \mathrm{Cq})$ of the target genes and the selected reference gene was calculated for each target gene; $\Delta \mathrm{Cq}_{\text {target }}=\mathrm{Cq}_{\text {target }}-\mathrm{Cq}_{\text {reference. }}$. An arbitrarily selected biological replicate of dL-0, aL- 0 and dcL- 0 was used as calibrator sample for the dorsal, anal and caudal fin, respectively. Hence, samples were normalized to the $\Delta \mathrm{Cq}$ value of the calibrator sample $\left(\Delta \mathrm{Cq}_{\text {target }}-\Delta \mathrm{Cq}_{\text {calibrator }}\right)$ to obtain a $\Delta \Delta \mathrm{Cq}$ value. Relative expression quantities (RQ) were calculated as $\mathrm{E}^{-\Delta \Delta \mathrm{Cq}}$ with $\mathrm{E}=2^{76}$. For each target gene, differences in expression levels (log-transformed RQ data) between L and S tissue, fins and developmental stages were tested in a mixed linear model with biological replicate as grouping factor (Supplementary data 2). In order to identify genes with consistent $\mathrm{L} / \mathrm{S}$ expression differences across fins and developmental stages, paired $\mathrm{t}$-tests were performed on log-transformed RQ data. To examine expression pattern similarities between the target genes, Pearson correlation coefficients $(r$ ) were calculated using R (http://www.r-project.org).

Ethical approval. All experimental protocols related to the fishes used in this study were approved by the Federal Ministry of Science, Research and Economy of Austria. Please identify the approving body and license numbers in the methods section.

Data availability. All the data represented in this study are provided within the main manuscript or in the supplementary materials.

\section{References}

1. Pfefferli, C. \& Jaźwińska, A. The art of fin regeneration in zebrafish. Regeneration 2, 72-83 (2015).

2. Nogueira, A. F. et al. Tetrapod limb and sarcopterygian fin regeneration share a core genetic programme. Nat. Commun. 7, 13364 (2016).

3. Nakamura, T., Gehrke, A. R., Lemberg, J., Szymaszek, J. \& Shubin, N. H. Digits and fin rays share common developmental histories. Nature 537, 225-228 (2016).

4. Nakatani, Y., Kawakami, A. \& Kudo, A. Cellular and molecular processes of regeneration, with special emphasis on fish fins. Dev. Growth Differ. 49, 145-154 (2007).

5. Marí-Beffa, M. \& Murciano, C. Dermoskeleton morphogenesis in zebrafish fins. Dev. Dyn. 239, 2779-2794 (2010).

6. Iovine, M. K. Conserved mechanisms regulate outgrowth in zebrafish fins. Nat. Chem. Biol. 3, 613-618 (2007).

7. Wehner, D. \& Weidinger, G. Signaling networks organizing regenerative growth of the zebrafish fin. Trends Genet. 31, 336-343 (2015).

8. Yoshinari, N., Ishida, T., Kudo, A. \& Kawakami, A. Gene expression and functional analysis of zebrafish larval fin fold regeneration. Dev. Biol. 325, 71-81 (2009).

9. Schebesta, M., Lien, C.-L., Engel, F. B. \& Keating, M. T. Transcriptional profiling of caudal fin regeneration in zebrafish. ScientificWorldJournal. 6(Suppl 1), 38-54 (2006).

10. Mathew, L. K. et al. Comparative expression profiling reveals an essential role for raldh2 in epimorphic regeneration. J. Biol. Chem. 284, 33642-53 (2009).

11. Kang, J. H. et al. Transcriptomics of two evolutionary novelties: how to make a sperm-transfer organ out of an anal fin and a sexually selected 'sword' out of a caudal fin. Ecol. Evol. 5, 848-864 (2015).

12. Nakamura, T. et al. Molecular mechanisms underlying the exceptional adaptations of batoid fins. Proc. Natl. Acad. Sci. USA 112, 15940-5 (2015).

13. Navon, D., Olearczyk, N. \& Albertson, R. C. Genetic and developmental basis for fin shape variation in African cichlid fishes. Mol. Ecol. 26, 291-303 (2017).

14. Abe, G. et al. The origin of the bifurcated axial skeletal system in the twin-tail goldfish. Nat. Commun. 5, 3360 (2014).

15. Ahi, E. P., Richter, F. \& Sefc, K. M. A gene expression study of ornamental fin shape in Neolamprologus brichardi, an African cichlid species. Sci. Rep. 7, 17398 (2017).

16. Iovine, M. K., Higgins, E. P., Hindes, A., Coblitz, B. \& Johnson, S. L. Mutations inconnexin43 (GJA1) perturb bone growth in zebrafish fins. Dev. Biol. 278, 208-219 (2005).

17. LeBert, D. C. et al. Matrix metalloproteinase 9 modulates collagen matrices and wound repair. Development 142, 2136-2146 (2015).

18. Ton, Q. V. \& Iovine, K. M. Semaphorin3d mediates Cx43-dependent phenotypes during fin regeneration. Dev. Biol. 366, 195-203 (2012).

19. Verlinden, L. et al. Nrp2 deficiency leads to trabecular bone loss and is accompanied by enhanced osteoclast and reduced osteoblast numbers. Bone 55, 465-75 (2013).

20. Ton, Q. V. \& Iovine, M. K. Identification of an evx1-Dependent Joint-Formation Pathway during FIN Regeneration. PLoS One 8, e81240 (2013).

21. Berndt, J. D. \& Halloran, M. C. Semaphorin 3d promotes cell proliferation and neural crest cell development downstream of TCF in the zebrafish hindbrain. Development 133, 3983-3992 (2006).

22. Sims, K., Eble, D. M. \& Iovine, M. K. Connexin43 regulates joint location in zebrafish fins. Dev. Biol. 327, 410-418 (2009).

23. Misu, A. et al. Two Different Functions of Connexin43 Confer Two Different Bone Phenotypes in Zebrafish. J. Biol. Chem. 291, 12601-12611 (2016)

24. De Bock, M., Wang, N., Decrock, E., Bultynck, G. \& Leybaert, L. Intracellular Cleavage of the Cx43 C-Terminal Domain by MatrixMetalloproteases: A Novel Contributor to Inflammation? Mediators Inflamm. 2015, 1-18 (2015).

25. Ahi, E. P. et al. Differential expression of the Aryl hydrocarbon receptor pathway associates with craniofacial polymorphism in sympatric Arctic charr. Evodevo 6, 27 (2015).

26. Obayashi, T. \& Kinoshita, K. COXPRESdb: a database to compare gene coexpression in seven model animals. Nucleic Acids Res. 39, D1016-22 (2011)

27. Davidson, E. H. \& Erwin, D. H. Gene regulatory networks and the evolution of animal body plans. Science 311, 796-800 (2006). 
28. Erwin, D. H. \& Davidson, E. H. The evolution of hierarchical gene regulatory networks. Nat. Rev. Genet. 10, 141-148 (2009).

29. Pfennig, D. W. \& Ehrenreich, I. M. Towards a gene regulatory network perspective on phenotypic plasticity, genetic accommodation and genetic assimilation. Mol. Ecol. 23, 4438-4440 (2014).

30. Filteau, M., Pavey, S. A., St-Cyr, J. \& Bernatchez, L. Gene Coexpression Networks Reveal Key Drivers of Phenotypic Divergence in Lake Whitefish. Mol. Biol. Evol. 30, 1384-1396 (2013).

31. Schneider, R. F., Li, Y., Meyer, A. \& Gunter, H. M. Regulatory gene networks that shape the development of adaptive phenotypic plasticity in a cichlid fish. Mol. Ecol. 23, 4511-4526 (2014).

32. Ahi, E. P. et al. Transcriptional dynamics of a conserved gene expression network associated with craniofacial divergence in Arctic charr. Evodevo 5, 40 (2014).

33. Kratochwil, C. F., Geissler, L., Irisarri, I. \& Meyer, A. \& S, K. Molecular Evolution of the Neural Crest Regulatory Network in RayFinned Fish. Genome Biol. Evol. 7, 3033-3046 (2015).

34. Banerji, R., Eble, D. M., Iovine, M. K. \& Skibbens, R. V. Esco2 regulates cx43 expression during skeletal regeneration in the zebrafish fin. Dev. Dyn. 245, 7-21 (2016).

35. Ahi, E. P. \& Sefc, K. M. Anterior-posterior gene expression differences in three Lake Malawi cichlid fishes with variation in body stripe orientation. PeerJ 5, e4080 (2017).

36. Saxena, S. et al. Role of annexin gene and its regulation during zebrafish caudal fin regeneration. Wound Repair Regen. 24, 551-559 (2016).

37. Nakatani, Y., Nishidate, M., Fujita, M., Kawakami, A. \& Kudo, A. Migration of mesenchymal cell fated to blastema is necessary for fish fin regeneration. Dev. Growth Differ. 50, 71-83 (2007).

38. Hagedorn, M., Siegfried, G., Hooks, K. B. \& Khatib, A.-M. Integration of zebrafish fin regeneration genes with expression data of human tumors in silico uncovers potential novel melanoma markers. Oncotarget 7, 71567-71579 (2016).

39. Andreasen, E. A., Mathew, L. K. \& Tanguay, R. L. Regenerative Growth Is Impacted by TCDD: Gene Expression Analysis Reveals Extracellular Matrix Modulation. Toxicol. Sci. 92, 254-269 (2006).

40. Ma, L., Yu, Y.-M., Guo, Y., Hart, R. P. \& Schachner, M. Cysteine- and glycine-rich protein la is involved in spinal cord regeneration in adult zebrafish. Eur. J. Neurosci. 35, 353-65 (2012).

41. Monaghan, J. R. et al. Early gene expression during natural spinal cord regeneration in the salamander Ambystoma mexicanum. $J$. Neurochem. 101, 27-40 (2006).

42. Lin, S. et al. Inhibition of Kinesin-5, a Microtubule-Based Motor Protein, As a Strategy for Enhancing Regeneration of Adult Axons. Traffic 12, 269-286 (2011).

43. Lehmann, O. J., Sowden, J. C., Carlsson, P., Jordan, T. \& Bhattacharya, S. S. Fox's in development and disease. Trends Genet. 19, 339-344 (2003).

44. Hannenhalli, S. \& Kaestner, K. H. The evolution of Fox genes and their role in development and disease. Nat. Rev. Genet. 10, 233-240 (2009).

45. Singh, A. M. \& Dalton, S. The Cell Cycle and Myc Intersect with Mechanisms that Regulate Pluripotency and Reprogramming. Cell Stem Cell 5, 141-149 (2009).

46. Nonchev, S. et al. The conserved role of Krox-20 in directing Hox gene expression during vertebrate hindbrain segmentation. Proc. Natl. Acad. Sci. USA 93, 9339-45 (1996).

47. Zaman, G. et al. Loading-related regulation of transcription factor EGR2/Krox-20 in bone cells is ERK1/2 protein-mediated and prostaglandin, Wnt signaling pathway-, and insulin-like growth factor-I axis-dependent. J. Biol. Chem. 287, 3946-62 (2012).

48. Kim, H.-J. et al. Early growth response 2 negatively modulates osteoclast differentiation through upregulation of Id helix-loop-helix proteins. Bone 51, 643-650 (2012)

49. Chandra, A., Lan, S., Zhu, J., Siclari, V. A. \& Qin, L. Epidermal growth factor receptor (EGFR) signaling promotes proliferation and survival in osteoprogenitors by increasing early growth response 2 (EGR2) expression. J. Biol. Chem. 288, 20488-98 (2013).

50. Alter-Koltunoff, M. et al. Innate immunity to intraphagosomal pathogens is mediated by interferon regulatory factor 8 (IRF-8) that stimulates the expression of macrophage-specific Nramp1 through antagonizing repression by c-Myc. J. Biol. Chem. 283, 2724-33 (2008).

51. Christen, B., Robles, V., Raya, M., Paramonov, I. \& Izpisúa Belmonte, J. C. Regeneration and reprogramming compared. BMC Biol. 8, 5 (2010).

52. Rao, N. et al. Proteomic analysis of blastema formation in regenerating axolotl limbs. BMC Biol. 7, 83 (2009).

53. Maki, N. et al. Expression of stem cell pluripotency factors during regeneration in newts. Dev. Dyn. 238, 1613-1616 (2009).

54. Smith-Bolton, R. K., Worley, M. I., Kanda, H. \& Hariharan, I. K. Regenerative Growth in Drosophila Imaginal Discs Is Regulated by Wingless and Myc. Dev. Cell 16, 797-809 (2009).

55. Li, L., Yan, B., Shi, Y.-Q., Zhang, W.-Q. \& Wen, Z.-L. Live imaging reveals differing roles of macrophages and neutrophils during zebrafish tail fin regeneration. J. Biol. Chem. 287, 25353-60 (2012).

56. Hasegawa, T. et al. Transient inflammatory response mediated by interleukin- $1 \beta$ is required for proper regeneration in zebrafish fin fold. Elife 6, e22716 (2017).

57. Petrie, T. A., Strand, N. S., Tsung-Yang, C., Rabinowitz, J. S. \& Moon, R. T. Macrophages modulate adult zebrafish tail fin regeneration. Development 141, 2581-2591 (2014).

58. Dror, N. et al. Identification of IRF-8 and IRF-1 target genes in activated macrophages. Mol. Immunol. 44, 338-346 (2007).

59. Cohen, S. B. et al. Beta-catenin signaling drives differentiation and proinflammatory function of IRF8-dependent dendritic cells. J. Immunol. 194, 210-22 (2015).

60. Scheller, M. et al. Cross talk between Wnt/3-catenin and Irf8 in leukemia progression and drug resistance. J. Exp. Med. 210, 2239-56 (2013).

61. Sauka-Spengler, T. \& Bronner-Fraser, M. A gene regulatory network orchestrates neural crest formation. Nat. Rev. Mol. Cell Biol. 9, 557-568 (2008).

62. Stewart, S. \& Stankunas, K. Limited dedifferentiation provides replacement tissue during zebrafish fin regeneration. Dev. Biol. 365, 339-49 (2012).

63. Stewart, S., Gomez, A. W., Armstrong, B. E., Henner, A. \& Stankunas, K. Sequential and Opposing Activities of Wnt and BMP Coordinate Zebrafish Bone Regeneration. Cell Rep. 6, 482-498 (2014).

64. Lewis, J. L. et al. Reiterated Wnt signaling during zebrafish neural crest development. Development 131, 1299-1308 (2004).

65. Huang, J. \& Chen, L. IL-1 $\beta$ inhibits osteogenesis of human bone marrow-derived mesenchymal stem cells by activating FoxD3/ microRNA-496 to repress wnt signaling. genesis 55, e23040, https://doi.org/10.1002/dvg.23040 (2017).

66. Wang, W.-D., Melville, D. B., Montero-Balaguer, M., Hatzopoulos, A. K. \& Knapik, E. W. Tfap2a and Foxd3 regulate early steps in the development of the neural crest progenitor population. Dev. Biol. 360, 173-185 (2011).

67. Gavriouchkina, D. et al. From pioneer to repressor: Bimodal foxd3 activity dynamically remodels neural crest regulatory landscape in vivo. bioRxiv 213611, https://doi.org/10.1101/213611 (2017).

68. Flicek, P. et al. Ensembl 2013. Nucleic Acids Res. 41, D48-55 (2013).

69. Bailey, T. L. et al. MEME SUITE: tools for motif discovery and searching. Nucleic Acids Res. 37, W202-8 (2009).

70. Luehr, S., Hartmann, H. \& Söding, J. The XXmotif web server for eXhaustive, weight matriX-based motif discovery in nucleotide sequences. Nucleic Acids Res. 40, W104-9 (2012).

71. Matys, V. et al. TRANSFAC: transcriptional regulation, from patterns to profiles. Nucleic Acids Res. 31, 374-8 (2003). 
72. Mahony, S. \& Benos, P. V. STAMP: a web tool for exploring DNA-binding motif similarities. Nucleic Acids Res. 35, W253-8 (2007).

73. Brawand, D. et al. The genomic substrate for adaptive radiation in African cichlid fish. Nature 513, 375-81 (2014).

74. Hellemans, J., Mortier, G., De Paepe, A., Speleman, F. \& Vandesompele, J. qBase relative quantification framework and software for management and automated analysis of real-time quantitative PCR data. Genome Biol. 8, R19 (2007).

75. Ramakers, C., Ruijter, J. M., Deprez, R. H. L. \& Moorman, A. F. M. Assumption-free analysis of quantitative real-time polymerase chain reaction (PCR) data. Neurosci. Lett. 339, 62-6 (2003).

76. Pfaffl, M. W. A new mathematical model for relative quantification in real-time RT-PCR. Nucleic Acids Res. 29, e45 (2001).

77. King, M. W., Neff, A. W. \& Mescher, A. L. Proteomics analysis of regenerating amphibian limbs: changes during the onset of regeneration. Int. J. Dev. Biol. 53, 955-969 (2009).

78. Pohl, B. S. \& Knöchel, W. Overexpression of the transcriptional repressor FoxD3 prevents neural crest formation in Xenopus embryos. Mech. Dev. 103, 93-106 (2001).

79. Bellmeyer, A., Krase, J., Lindgren, J. \& LaBonne, C. The Protooncogene c-Myc Is an Essential Regulator of Neural Crest Formation in Xenopus. Dev. Cell 4, 827-839 (2003).

80. Boone, D. N., Qi, Y., Li, Z. \& Hann, S. R. Egr1 mediates p53-independent c-Myc-induced apoptosis via a noncanonical ARFdependent transcriptional mechanism. Proc. Natl. Acad. Sci. USA 108, 632-7 (2011).

81. Zhen, Y. CardioTF, a database of deconstructing transcriptional circuits in the heart system. PeerJ 4, e2339 (2016).

82. Zhu, H. Forkhead box transcription factors in embryonic heart development and congenital heart disease. Life Sci. 144, 194-201 (2016).

83. Wang, L., Siegenthaler, J. A., Dowell, R. D. \& Yi, R. Foxc1 reinforces quiescence in self-renewing hair follicle stem cells. Science 351, 613-7 (2016)

\section{Acknowledgements}

The authors thank Wolfgang Gessl (www.pisces.at) for his responsible management of our fish facility and photography of the intact and regenerating fish fins. We also thank Holger Zimmermann and Stephan Koblmüller for sharing their precious knowledge on cichlid fishes of Lake Tanganyika, as well as Silke Werth and the Institute of Plant Sciences at University of Graz for technical assistance and access to their real-time PCR System. The authors acknowledge the financial support by the University of Graz.

\section{Author Contributions}

E.P.A. and K.M.S. designed the study, analysed the expression data and wrote the manuscript. E.P.A. has conducted the laboratory experiment and figure preparations for gene expression sections. Both authors reviewed the manuscript and approve its content.

\section{Additional Information}

Supplementary information accompanies this paper at https://doi.org/10.1038/s41598-018-27977-y.

Competing Interests: The authors declare no competing interests.

Publisher's note: Springer Nature remains neutral with regard to jurisdictional claims in published maps and institutional affiliations.

(c) (i) Open Access This article is licensed under a Creative Commons Attribution 4.0 International License, which permits use, sharing, adaptation, distribution and reproduction in any medium or format, as long as you give appropriate credit to the original author(s) and the source, provide a link to the Creative Commons license, and indicate if changes were made. The images or other third party material in this article are included in the article's Creative Commons license, unless indicated otherwise in a credit line to the material. If material is not included in the article's Creative Commons license and your intended use is not permitted by statutory regulation or exceeds the permitted use, you will need to obtain permission directly from the copyright holder. To view a copy of this license, visit http://creativecommons.org/licenses/by/4.0/.

(c) The Author(s) 2018 